\title{
Primary sternal tuberculosis osteomyelitis: A case report and discussion
}

\author{
Miten Vasa $M D^{1}$, Christine Ohikhuare MD $^{2}$, Leslea Brickner MD FACP ${ }^{3}$
}

M Vasa, C Ohikhuare, L Brickner. Primary sternal tuberculosis osteomyelitis: A case report and discussion. Can J Infect Dis Med Microbiol 2009;20(4):e181-e184.

As immigration to the United States from countries endemic for tuberculosis (TB) increases, the incidence of pulmonary and extrapulmonary TB disease may increase. Primary tuberculous sternal osteomyelitis is one form of extrapulmonary $\mathrm{TB}$ that is exceedingly rare throughout the world, and falls under the differential diagnosis for chest wall masses. Management involves standard antituberculous therapy with antibiotics similar to treating other forms of extrapulmonary $\mathrm{TB}$, as well as consideration of surgical intervention depending on the extent of osteomyelitis. A typical case of primary sternal TB osteomyelitis is reported, and the epidemiology, differential diagnosis, clinical manifestations and management are reviewed.

Key Words: Osteomyelitis; Primary; Review; Sternal; Sternum; Tuberculosis

\section{L'ostéomyélite tuberculeuse sternale primaire : Rapport de cas et exposé}

Tandis que l'immigration aux États-Unis en provenance de pays endémiques à la tuberculose (TB) augmente, l'incidence de TB pulmonaire et extrapulmonaire pourrait augmenter. L'ostéomyélite tuberculeuse sternale primaire est une forme de TB extrapulmonaire d'une extrême rareté dans le monde, et elle fait partie du diagnostic différentiel des masses de la paroi thoracique. La prise en charge inclut une thérapie antituberculeuse aux antibiotiques normale, similaire au traitement d'autres formes de TB extrapulmonaire, ainsi qu'une possibilité d'intervention chirurgicale selon l'étendue de l'ostéomyélite. Les auteurs présentent un cas classique d'ostéomyélite tuberculeuse sternale primaire et passent en revue l'épidémiologie, le diagnostic différentiel, les manifestations cliniques et la prise en charge de la maladie.
$\mathrm{T}$ uberculosis (TB) continues to be a major international problem, particularly in less developed countries, with an estimated global case fatality rate of $13 \%$ in 2007 . The World Health Organization estimated that there were 13.7 million prevalent cases of TB infection worldwide, with each year bringing about 9.27 million new cases, $44 \%$ of which are new smear-positive cases (1). The total number of global cases is still increasing in absolute terms as a result of population growth, but the number of incident cases per capita is declining. Despite reductions in the global burden of TB, 37\% of smear-positive TB cases are not being treated, more than $90 \%$ of multidrug resistant TB (MDR-TB) are not being diagnosed and treated, the majority of HIV-positive TB patients do not know their HIV status, and those who do are not yet accessing antiretroviral therapy (1).

Since 1985, there has been an increase in the incidence of TB per capita, TB prevalence and TB mortality worldwide, which peaked in 2004, 1990 and 2000, respectively, and is now trending down due to international efforts at disease control. However, the increased incidence in certain countries could be attributed to immunosuppression (HIV, aging populations), drug-resistant strains of mycobacterium, an increase in the number of health care workers exposed to disease, immigration from endemic regions and new data available from a larger number of countries $(1,2)$.

The World Health Organization regions of Southeast Asia and the Western Pacific accounted for 55\% of global TB cases, and the African Region accounted for $31 \%$ in 2007. In contrast, the Americas, Europe and Eastern Mediterranean regions account for small fractions of global cases (1). The Philippines, where our patient is from, has the seventh highest TB burden among all countries in the world as of 2007 (1). MDR-TB cases have also been rising throughout the world, with approximately $4.9 \%$ of new cases ( 0.3 million cases) and $19 \%$ of previously treated TB cases ( 0.2 million cases) having MDR-TB in 2007. The Philippines ranks eighth in the world in the number of MDR-TB cases, with $4 \%$ of new cases and $21 \%$ of previously treated TB cases having MDR-TB (1).

TB can disseminate hematogenously to almost every organ after a primary infection or reactivation of latent foci, occurring more commonly in an immunodeficient patient. The pathogenesis and source of latent foci and subsequent routes of dissemination are incompletely understood, but three reported theories include spread as a late complication of pulmonary tuberculosis, reactivation of latent foci formed during hematogenous or lymphatic dissemination of primary tuberculosis, or direct extension from mediastinal lymph nodes (3). Extrapulmonary TB has been particularly prominent since 1985 , accounting for approximately $17.5 \%$ to $32 \%$ of all TB cases in the United States, with approximately $6 \%$ of these synchronous with pulmonary TB $(4,5)$. Skeletal TB accounts for approximately $6 \%$ to $10 \%$ of extrapulmonary TB cases and $1 \%$ of all TB cases in the United States (4,6-8).

Primary sternal osteomyelitis accounts for approximately $0.3 \%$ of all types of osteomyelitis (9). Approximately $60 \%$ to $80 \%$ of skeletal TB cases involve the spine or weight-bearing joints, while the sternum is involved in approximately $1 \%$ of cases $(8,10,11)$. Primary tuberculous sternal osteomyelitis is even more uncommon. Thus, diagnosis of sternal TB is often not considered, particularly in developed countries. Often the diagnosis is not made until the disease is well advanced, with only about 25 cases reported in peer-reviewed journals in the post-antituberculous therapy (ATT) era in our literature review (12).

Department of Internal Medicine, Oakland Kaiser Permanente Department of Medicine, Oakland, California, USA

Correspondence: Dr Miten Vasa, Kaiser Permanente Oakland Medical Center, Department of Internal Medicine, 280 West MacArthur

Boulevard, Oakland, California 94611, USA. Telephone 516-286-1260, e-mail Miten.X.Vasa@kp.org or miten.vasa@gmail.com 


\section{CASE PRESENTATION}

A case of primary sternal TB osteomyelitis in a 55-year-old Filipino man is presented. He experienced nonspecific neck and shoulder pain for 2.5 years before admission to the hospital when the diagnosis was finally made.

The patient had been treated two years before diagnosis in the Philippines for 'chest swelling' that completely resolved after two weeks of antibiotics. He moved to the United States and was seen in clinic with musculoskeletal symptoms 15 months, three months and two months before diagnosis. At the first clinic visit, he reported tight, painful muscles in the posterior neck and shoulders for several months, with tender bilateral trapezius muscles. He denied any trauma or prior operative procedures, and was given ibuprofen for a suspected trapezius muscle strain. At the second clinic visit one year later, he presented with one week of chest and upper back pain, with chest tenderness that was unrelieved by acetaminophen or ibuprofen. His electrocardiogram was normal, and he was prescribed nabumetone for suspected costochondritis. At the third clinic visit, one month later, the patient reported the same musculoskeletal complaints as well as new chest swelling, which was noted on physical examination. He had tenderness at the sternoclavicular areas bilaterally, as well as tenderness of the manubrium, anterior and posterior neck, and trapezius. $\mathrm{He}$ also had limited range of motion of his neck and bilateral upper extremities due to pain. The patient denied any constitutional symptoms. He had just come back from a three-week vacation in the Philippines, but was experiencing these symptoms before his trip. Posteroanterior and lateral chest radiographs and clavicular plain films were normal. The patient was prescribed hydrocodone/acetaminophen along with nabumetone for musculoskeletal chest pain.

Two weeks later, the patient returned with increased chest swelling and erythema, and new headache, night sweats, nausea and cough. His vital signs were significant for a temperature of $37.4^{\circ} \mathrm{C}$. He had an $8 \mathrm{~cm} \times 10 \mathrm{~cm}$ nonfluctuant, erythematous mass, raised approximately $1 \mathrm{~cm}$, in the manubrial area that was warm and tender, but without any drainage (Figure 1). He also had right-sided cervical lymphadenopathy, and decreased range of motion of his neck and bilateral upper extremities. Laboratory results revealed leukocytosis $\left(20 \times 10^{9} / \mathrm{L}\right.$ white blood cells with $81 \%$ neutrophils), and an elevated erythrocyte sedimentation rate at $88 \mathrm{~mm} / \mathrm{h}$ and C-reactive protein level at $12.4 \mathrm{mg} / \mathrm{dL}$. Based on the patient's overall clinical picture, a computed tomography (CT) scan of the chest was ordered to rule out an abscess, tumour or phlegmon.

CT scan of the chest demonstrated upper sternal anterior and posterior osteomyelitis (Figure 2) along with bilateral adjacent costosternal abscesses and a mildly enlarged right supraclavicular lymph node. There was no evidence of lung disease, and an HIV test was negative. The patient was started on vancomycin and piperacillin-tazobactam empirically in the hospital for broad antimicrobial coverage for osteomyelitis, and he also had a tuberculin skin test placed on admission. Initially it was believed that the abscesses were too small to drain or aspirate, and that an invasive biopsy could be avoided if the patient responded to antibiotics alone. However, because the patient was not improving, a needle aspiration of the sternum was performed and sent for Gram stain, aerobic, anaerobic, fungal and acid-fast bacilli cultures, and cytology. At this time, his tuberculin skin test was positive at $20 \mathrm{~mm}$ of induration. $\mathrm{He}$ had never received a bacille Calmette-Guérin (BCG) vaccine, but he had spent most of his life in the Philippines. BecauseTB was the more likely etiology, vancomycin and piperacillintazobactam were stopped and ATT was started empirically (rifampin $600 \mathrm{mg} /$ day, pyrazinamide $2000 \mathrm{mg} /$ day, ethambutol $1600 \mathrm{mg} /$ day and isoniazid $300 \mathrm{mg} /$ day), although there was concern about resistant TB given that the patient had received antibiotics before and was from a country endemic for $\mathrm{TB}$ where resistance can be seen. Biopsy results of the sternum subsequently showed acid-fast bacilli, and a nucleic acid probe was positive for Mycobacterium tuberculosis complex. The needle aspiration culture came back positive for $\mathrm{M}$ tuberculosis, which was sensitive to all four ATT drugs. All other cultures and cytology were negative, and acid-fast bacilli sputum cultures were negative. A white blood cell scan and magnetic resonance imaging (MRI) of the spine showed no other skeletal or soft tissue involvement, and confirmed the CT findings. A cardiothoracic surgery consult report stated that the patient's sternum was stable, and recommended continuing management with ATT alone. If the patient's condition declined or failed to improve they would consider resection of the upper sternum, followed by a pectoralis muscle flap closure by plastic surgery. The patient was discharged from the hospital one week after admission on ATT after counselling, education and approval by the county's Department of Public Health. The county also provided the patient with directly observed therapy via a visiting nurse. At follow-up three and six months later, the patient had significant improvement in his sternal swelling and erythema and was continued on isoniazid and rifampin. He denied any constitutional symptoms, draining sinuses, sternal erythema or pain. His chest pain, neck pain and range of motion have greatly improved.

\section{DISCUSSION}

Sternal TB osteomyelitis presents with clinical manifestations similar to other forms of osteoarticular TB disease, including soft tissue swelling, which is the most common symptom, seen in $81 \%$ of patients with sternal TB; bone pain and swelling; erythema, warmth and tenderness; enlarged regional lymph nodes; bone deformity or fracture; or a draining abscess or sinus. Constitutional symptoms are less commonly seen, but include malaise, fever, night sweats or weight loss. As in our patient, clinical manifestations of sternal TB present in an indolent manner before diagnosis, with a mean of nine to 13 months, and a range of two months to six years before diagnosis. Also, there appears to be a male predominance, with a mean age of 36 years (range 11 to 59 years). Inflammatory markers such as erythrocyte sedimentation rate, C-reactive protein lactate dehydrogenase and white blood cell count are almost universally elevated $(13,14)$.

Thoracic TB disease most commonly involves the shafts of the ribs or the costovertebral or costochondral junctions. The proportion of sternal involvement of all thoracic TB cases in series from England, India, and Saudi Arabia ranges from 0\% to 7\% $(13,14)$. More than $70 \%$ of cases of sternal TB osteomyelitis have an abnormal tuberculin skin test result. Chest radiographs are normal in approximately $70 \%$ of these cases, and approximately $40 \%$ have evidence of TB in sites other than the sternum, with the lymphatic system being the most common $(13,14)$. 


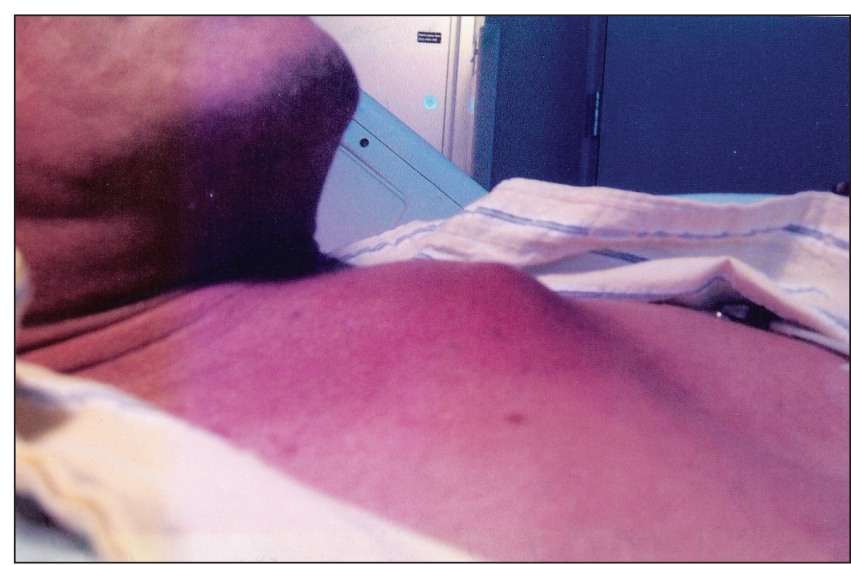

Figure 1) Sternal tuberculosis osteomyelitis presenting as chest wall swelling and erythema

Risk markers for TB infection include a positive tuberculin skin test, immunodeficiency (ie, HIV-positive, elderly), high risk for exposure to TB (ie, endemic countries, health care workers, low socioeconomic level), recent BCG vaccination and a history of intravenous drug abuse $(7,15)$. A recent sternotomy wound after cardiovascular surgery is also associated with sternal TB, presumably from reactivation of pulmonary $\mathrm{TB}$, but this is a rare complication with only five cases reported in the English literature from 1970 to 2006 (16). Interestingly, receipt of the BCG vaccine within the previous year has been associated with sternal osteomyelitis as a rare complication of the vaccine, presenting similarly to primary sternal TB osteomyelitis. Biopsy shows caseous necrosis and granulomatous inflammation, but cultures will be negative for TB in more than one-half of these rare cases. Sternal osteomyelitis as a complication of the BCG vaccine has been noted to resolve after treatment with antituberculous medications $(7,17)$.

The differential diagnosis of chest wall masses with or without discharging sinuses includes pyogenic infections (ie, Staphylococcus or Streptococcus species), malignancy (lymphoma or metastatic breast, lung or prostate cancer), Brodie's abscess and granulomatous lesions (sarcoidosis, Mycobacterium species, or fungal infections from Coccidioides, Histoplasma, Blastomyces or Cryptococcus) $(12,15,18)$. Pyogenic sternal infections are more fulminant due to proteolytic enzymes that can destroy cartilage, and often present with prominent constitutional symptoms. In contrast, TB does not produce proteolytic enzymes and cartilage is first destroyed peripherally, preserving the joint space for a considerable length of time. Thus, TB of the sternum can present with an indolent, painless course without constitutional symptoms, or a more aggressive course causing painful bone destruction with constitutional symptoms $(3,6)$. In Martini and Cuahes' case series $(10)$, they found $81 \%$ of TB osteomyelitis cases had a sinus formation and $43 \%$ of these had a superadded pyogenic infection, which was usually caused by Staphylococcus aureus. This superadded infection could lead to misdiagnosis, and could explain the more aggressive phase of the disease (10).

Bone pain that does not respond to analgesic medications is characteristic of an infection like TB or a neoplasm, so appropriate imaging with CT or MRI should be pursued when plain radiographs are normal (15). Clinical symptoms may precede radiological changes, which are varied depending

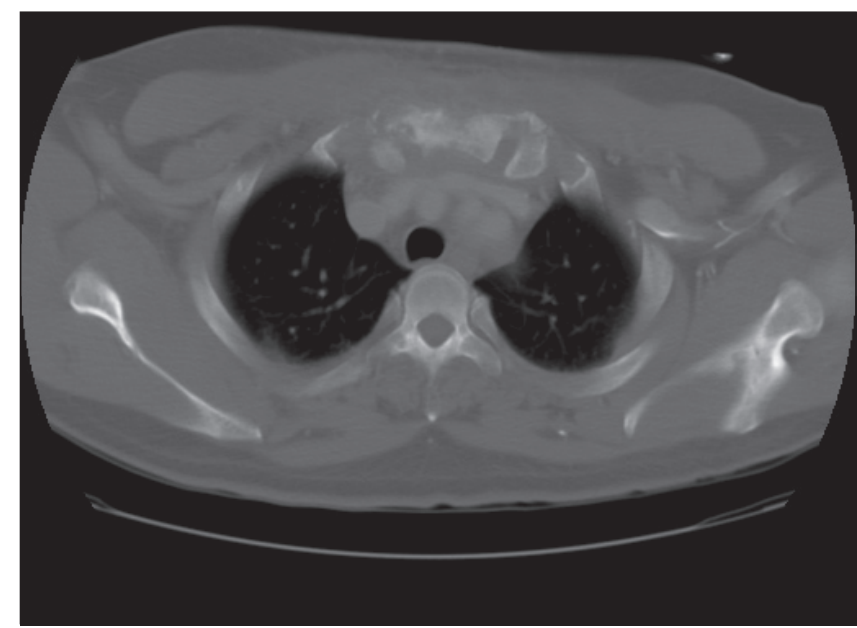

Figure 2) Computed tomography scan showing mixed sclerosis and lucency in a heterogenous hypodense soft tissue mass in the upper sternum with indistinct margins exhibiting peripheral enhancement consistent with osteomyelitis anterior and posterior to the sternum

on the progression of osteomyelitis and include osteoporosis, osteopenia, bone lysis, sclerosis, periostitis or pathological fracture. Plain radiographs are often normal, but CT shows the extent of bone and joint destruction. MRI delineates abscesses better in the soft tissues, and highlights bone marrow involvement. A three phase bone scan has high sensitivity and specificity for osteomyelitis, but gives less anatomic detail and does not indicate the cause of infection $(3,14,19)$. Gallium scans can be useful in showing other involved organs, but are also nonspecific with regard to the cause of infection and extent of damage (20).

A needle aspiration or excisional biopsy is mandatory for histopathological diagnosis of sternal osteomyelitis, because radiological findings cannot differentiate the cause of osteomyelitis, and sometimes may even appear neoplastic. The frequency of positive cultures is similar between both types of biopsies with approximately $85 \%$ showing acid-fast bacilli, but excisional biopsies are more likely to demonstrate granulomata and acid-fast bacilli. The biopsy or aspirate can be soft or friable, with whitish-grey debris resembling clumped cheesy material typical for caseous necrosis, which is very suggestive of TB. This was not seen in our particular case. Polymerase chain reaction and nucleic acid probes are now available for more rapid identification of mycobacteria not readily seen by microscope. Polymerase chain reaction for $\mathrm{M}$ tuberculosis complex is a rapid test that shows good correlation with histological findings, with an $85 \%$ sensitivity and $80 \%$ specificity. Mycobacterial sensitivities on cultures should always be done due to the increasing incidence of $\operatorname{MDR}-\mathrm{TB}(14,16)$.

ATT is the mainstay of treatment for sternal TB osteomyelitis currently. Although there is no consensus guideline to the precise regimen and duration for sternal $\mathrm{TB}$, extrapulmonary TB is generally treated with a six to nine month regimen (two months of isoniazid, rifampin, pyrazinamide and ethambutol, followed by four to seven months of isoniazid and rifampin), unless the organisms are known or highly suspected to be resistant to these first-line drugs (21). Extended therapy may be required for osteoarticular TB disease, delayed treatment response or drug resistance. Directly observed therapy is 
strongly recommended to better ensure medication compliance $(5,22)$. According to various published regimens, treatment can be continued up to 1.5 years after the first signs of clinical response, which can be seen in the first two to six months for sternal TB disease. These regimens consist of combinations of rifampin, isoniazid, pyrazinamide and ethambutol for the first two to seven months, followed by maintenance therapy with isoniazid and rifampin $(3,5,13,14)$. Variations in regimens depend on clinical response, side effects, and culture and sensitivity results. Clinical response precedes radiographic response, and the pain often resolves anywhere from two to six months before radiographic findings (3).

Many authors report surgical debridement to be essential to prevent recurrence or formation of a draining sinus $(19,23,24)$. They believe early drainage and complete debridement of necrotic material, which may include sternectomy, concomitant with ATT should be the mainstay of treatment; however, there are no formally defined surgical indications in the literature. Lesions of the sternal body are more common than of the manubrium. Lesions of the sternal body can be complicated by problems with skin closure, pectus excavatum or secondary mediastinal spread whereas manubrial involvement can be complicated by spread to the sternoclavicular joints. Debridement can be followed by rotational tissue flaps (pectoralis major, rectus abdominis, latissiums dorsi or omentum flap closure) and/or vacuum-assisted closure to cover the chest wall defect and prevent chronic sinus formation and the need for secondary reconstructive procedures. Secondary mediastinitis and wound dehiscence are potential complications that may require sternectomy and major chest wall reconstruction

\section{REFERENCES:}

1. World Health Organization. Global tuberculosis control epidemiology, strategy, financing: WHO Report 2009. <http://www. who.int/tb/publications/global_report/2009/en/index.html>

2. Dye C, Scheele S, Dolin P, et al. Global burden of tuberculosis: Estimated incidence, prevalence, and mortality by country. JAMA 1999;282:677-86.

3. Khan SA, Varshney MK, Hasan AS, et al. Tuberculosis of the sternum: A clinical study. J Bone Joint Surg Br 2007;89:817-20.

4. Rieder HL, Snider DE, Cauthen GM. Extrapulmonary tuberculosis in the United States. Am Rev Respir Dis 1990;141:347-51.

5. Cohn DL, Catlin BJ, Peterson KL, Judson FN, Sbarbaro JA. A 62-dose, 6-month therapy for pulmonary tuberculosis and extrapulmonary tuberculosis. A twice-weekly, directly observed and cost-effective regimen. Ann Intern Med 1990;112:407-15.

6. Davidson PT, Horowitz I. Skeletal tuberculosis: A review with patient presentations and discussion. Am J Med 1970;48;77-84.

7. Aribas OK, Kanat F, Gormus N, et al. Cold abscess of the chest wall as an unusual complication of BCG vaccination. Eur J Cardiothorac Surg 2002;21:352-4.

8. Zhao X, Chen S, Deanda A, et al. A rare presentation of tuberculosis. Am Surg 2006;72:96-7.

9. Maier HC. Infection of the costal cartilage and sternum. Surg Gynecol Obstet 1947;84:1038-44.

10. Martini M, Cuahes M. Bone and joint tuberculosis: A review of 652 cases. Orthopedics 1988;11:861-6.

11. Davies PD, Humphries MJ, Byfield SP, et al. Bone and joint tuberculosis: A survey of notifications in England and Wales. J Bone Joint Surg Br 1984;66:326-30.

12. Bajracharya, S, Singh GK, Singh M. Primary tubercular osteomyelitis of the sternum: Report of two cases. Internet J Third World Med 2007; 5(2). <www.ispub.com/journal/the_internet_ journal_of_third_world_medicine.html >

13. Dhillon MS, Gupta RK, Bahadur R, et al. Tuberculosis of the sternoclavicular joints. Acta Orthopaedica 2001;72:514-7.
$(3,19)$. Interestingly, a similar approach to therapy for other types of mycobacterial osteomyelitis (Mycobacterium ulcerans) involving surgical debridement and six months of combination antibiotics has proven successful, as well (24).

However, Khan et al (3) found that in their series of 14 patients followed for a mean of 2.8 years, surgical intervention was only necessary for the following: need for an open biopsy if needle aspiration is inconclusive, draining sinuses, debridement to promote early healing for markedly damaged or sequestrated bones or joint on radiographs such as pectus excavatum, wound dehiscence, extensive mediastinal disease or worsening disease, signs of secondary infection or mediastinitis, or disease not responding to an effective course of antibiotics (approximately two to six months of ATT) (3). Other authors have stated similar indications for surgery, but a larger study with longer follow-up will be needed to clearly establish these indications.

\section{CONCLUSION}

Our patient demonstrated a classic presentation of an extremely rare manifestation of tuberculosis that is not usually seen in the United States. As always, a proper history and physical examination are the keys to forming an appropriate differential diagnosis. Despite sternal TB's long, indolent course and ability to cause extensive osteomyelitis, ATT alone has succeeded in relieving the present patient's symptoms and he will likely not need surgery.

DISCLOSURE: All authors have participated in the research, and have reviewed and agree with the content of the article. There were no conflicts of interest, and no funding.

14. McLellan DG, Philips KB, Corbett CE, et al. Sternal osteomyelitis caused by mycobacterium tuberculosis: Case report and review of the literature. Am J Med Sci 2000;319:250-4.

15. Vohra R, Kang HS, Dogra S, et al. Tuberculous osteomyelitis. J Bone Joint Surg Br 1997;79:562-6.

16. Wang TK, Wong C, Au W, et al. Mycobacterium tuberculosis sternal wound infection after open heart surgery: A case report and review of the literature. Diagn Microbiol Infect Dis 2007;58:245-9.

17. Kato Y, Horikawa Y, Nishimura Y, et al. Sternal tuberculosis in a 9-month-old infant after BCG vaccination. Acta Paediatr 2000;89:1495-9.

18. Bohl JM, Janner D. Mycobacterium tuberculosis sternal osteomyeltis presenting as anterior chest wall mass. Pediatr Infect Dis J 1999;18:1028-9.

19. Ford SJ, Rathinam S, King JE, et al. Tuberculous osteomyelitis of the sternum: Successful management with debridement and vacuum assisted closure. Eur J Cardiothorac Surg 2005;28:645-7.

20. Choi H, Lee CJ, Lee KJ, et al. Primary tuberculous osteomyelitis of the sternum. J Cardiovasc Surg (Torino) 2001;42:841-3.

21. American Thoracic Society; CDC; Infectious Diseases Society of America. Treatment of tuberculosis MMWR Recomm Rep 200320;52(RR-11):1-77. (Erratum in 2005;53:1203).

22. Blumberg HM, Leonard MK Jr, Jasmer RM. Update on the treatment of tuberculosis and latent tuberculosis infection. JAMA 2005;293:2776-84.

23. Hsu HS, Wang LS, Wu YC, et al. Management of chest wall tuberculosis. Scand J Thorac Cardiovasc Surg 1995;29:119-23.

24. O'Brien DP, Athan E, Hughes A, et al. Successful treatment of Mycobacterium ulcerans osteomyelitis with minor surgical debridement and prolonged rifampicin and ciprofloxacin therapy: A case report. J Med Case Rep 2008;2:123. 


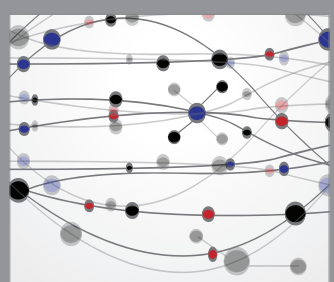

The Scientific World Journal
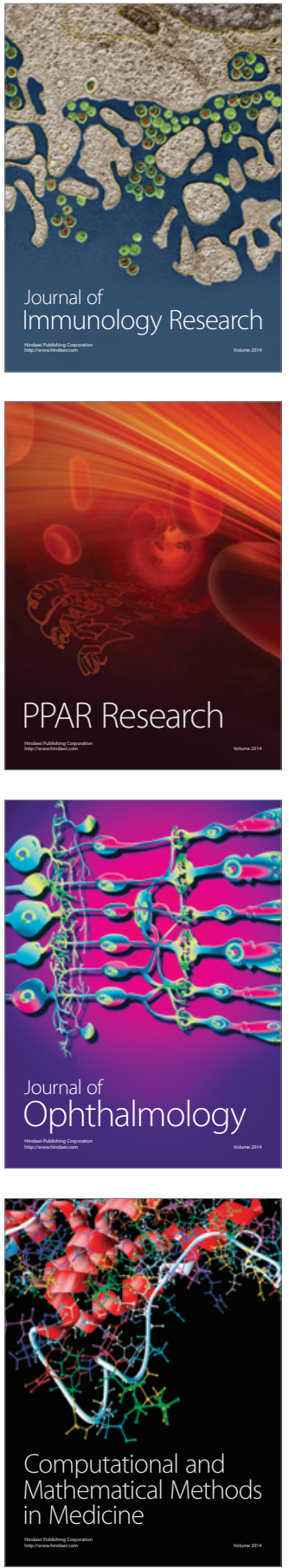

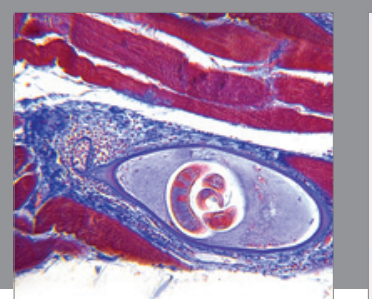

Gastroenterology Research and Practice

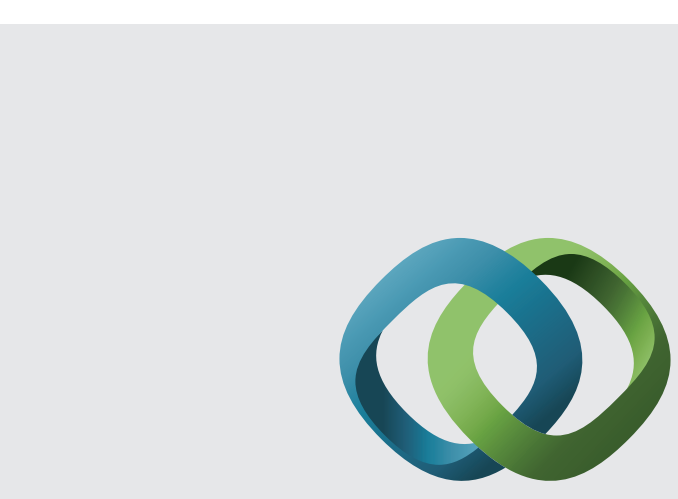

\section{Hindawi}

Submit your manuscripts at

http://www.hindawi.com
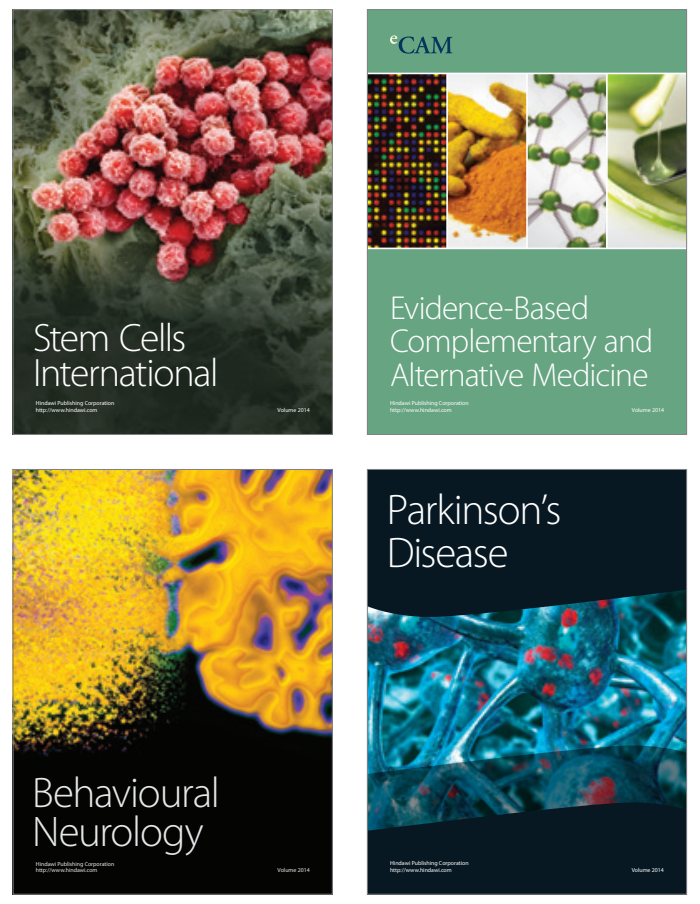
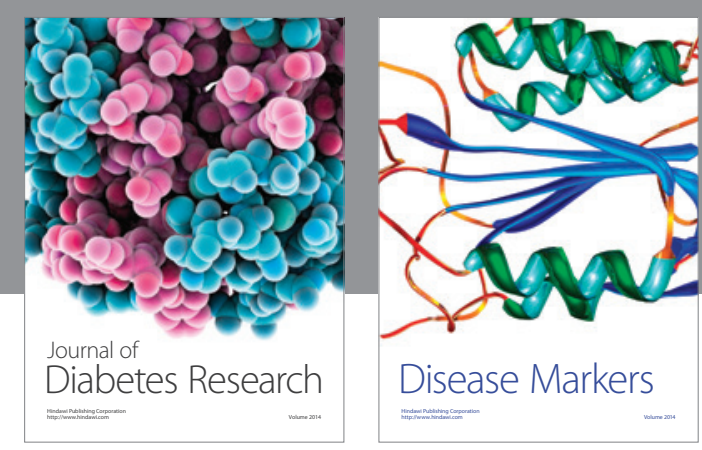

Disease Markers
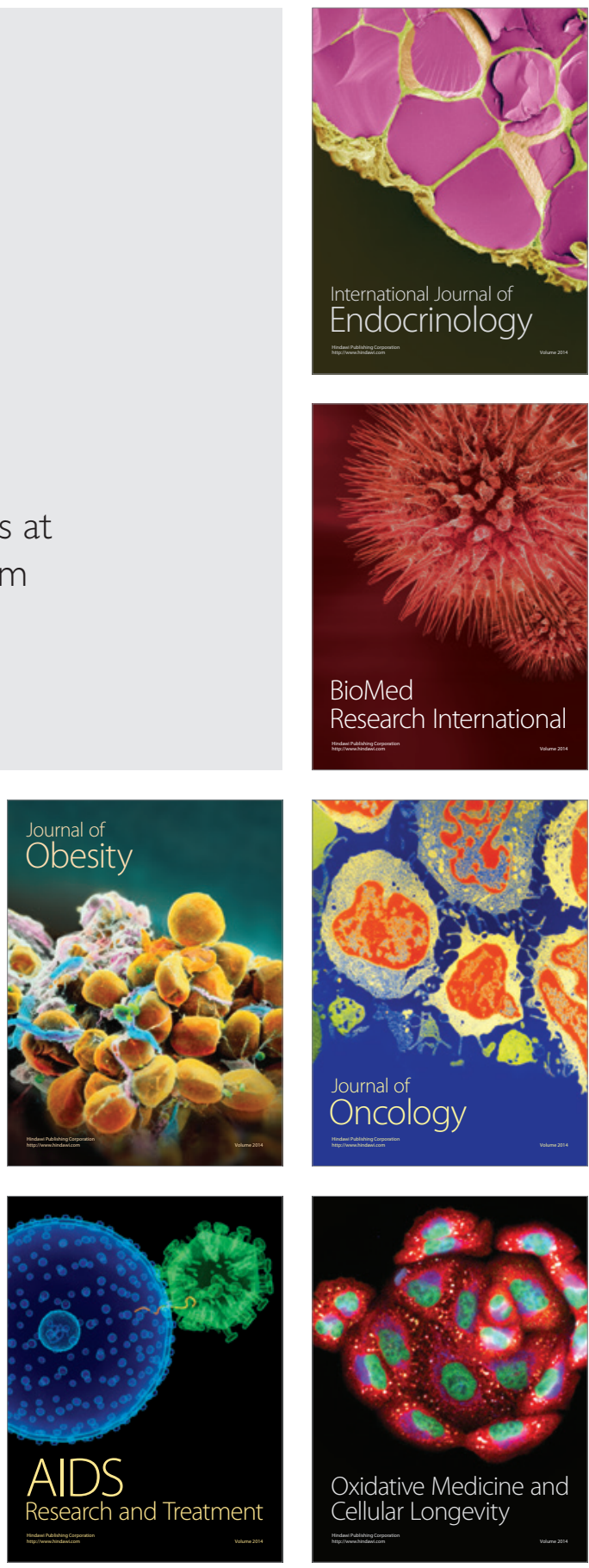